\title{
Sleep loss and sleep disorders
}

\section{Shedding light on common but under-recognised individual and community problems}

Darren R Mansfield MB BS, FRACP Deputy Director

David R Hillman MB BS, FANZCA, FRCPE, Sleep Physician ${ }^{2}$

Nicholas A Antic MB BS, FRACP, PhD, Clinical Director and Sleep and Respiratory Physician ${ }^{3,4}$

R Doug McEvoy

MB BS, FRACP

Senior Director, ${ }^{3}$ and Professor of Medicine

Shantha M W

Rajaratnam

Professor

1 Monash Respiratory and Sleep Medicine Monash Health

Melbourne, VIC.

2 Department of

Pulmonary Physiology and Sleep Medicine

Sir Charles Gairdner

Hospital, Perth, WA.

3 Adelaide Institute for Sleep Health

Repatriation General

Hospital, Adelaide, SA

4 Flinders University Adelaide, SA.

5 School of Psychology and Psychiatry,

Monash University,
Melbourne, VIC

darren.mansfield@ southernhealth.org.au

MJA 2013; 199: S5-S6 doi: 10.5694/mjal3.11157

Online first 17/10/13 $\mathrm{y}$ the time the average person reaches his or her average life expectancy of around 80 years, they will have invested 28 years of their lives in sleep. It is remarkable that an activity of this scale is so taken for granted. Ironically, it is the defining characteristic of sleep - perceptual disengagement from the environment that may provide the explanation for our disinclination to give our need for sleep its due attention. There is a natural tendency to invest effort in activities that provide conscious reward, and sleep risks being assigned a low priority compared with activities that occur during wakefulness. Importantly, these wakeful activities suffer where sleep is impaired.

While there are numerous hypotheses regarding the precise purpose of sleep, much of what we understand comes from experimental and naturalistic studies of individuals who are subjected to, or subject themselves to, inadequate sleep. Chronic sleep deficiency is believed to be widespread in Western societies. ${ }^{1}$ Sleep deficiency adversely affects alertness, cognition, productivity, safety, learning and mood and is implicated in a raft of additional pathophysiological processes, leading to adverse metabolic, cardiovascular and mental health outcomes, and premature death. This demands programs to improve sleep habits of the community generally and to detect and treat sleep disorders where they exist.

Linking sleep loss to specific adverse physiological and psychological consequences has led to some important, although limited, instances of behavioural change at the community level. Useful examples can be found in safetycritical industries, where alertness failure can have significant and potentially catastrophic consequences. Transport, aviation and, to some extent, health care industries have attempted to improve rostering practices to allow adequate opportunity for sleep and to minimise disruption of endogenous circadian pacemaker sleep-wake cycles. The duty to provide a safe work environment through enlightened roster arrangements rests with the employer. Equally importantly, employees should act on opportunities for sleep afforded by these rostering systems to ensure that they are "fit for duty". Although as much as 16\% of the Australian workforce is employed in shift work, optimal rostering systems and evidence-based countermeasures for workplace sleepiness are not always readily available or implemented. Despite some progress, the consequences of sleep loss remain under-recognised across the majority of workplaces and much of the community. The direct relationships between healthy sleep and a healthier, more productive and safer community needs to be better understood.

The previous Labor government in Australia identified productivity and population health and wellbeing as strategic research priorities, and as being among the most important challenges facing Australia (http://www.innovation.gov.au/research/Documents/SRP_fact_sheet_
WEB.PDF). Good sleep health is a core consideration in both these domains. However, there remains insufficient awareness at leadership level of the importance of optimal sleep in achieving these national goals. The issue of sleep health is yet to be addressed in our national preventive health strategy. Diet and exercise are regarded as key components of a healthy lifestyle, yet despite compelling evidence, minimal attention has been given to healthy sleep. Sleep medicine and sleep science, which are relatively new fields, have much to do to deliver this message to community leaders.

As much as improvement is required in existing practices, sleep health is also confronted by new and emerging challenges. Communities are faced with a rate of technological advancement that is historically unprecedented. The extent to which technology intrudes on our sleep routines remains incompletely defined. However, current observational evidence implicates electronic entertainment and communication devices in sleep loss, academic underachievement and obesity among adolescents. ${ }^{2}$ Further, late night exposure to the light that is emitted from these devices may disrupt the circadian pacemaker, compounding the sleep disturbance and potential health consequences. There is an urgent need to better educate adolescents and their parents about optimal sleep routines. Schools could be powerful allies in this effort.

As much as unhealthy sleep habits are pervasive in modern society, it is critical that the discipline of sleep medicine ensures that community efforts are focused on effective and cost-effective solutions. Sustainability of any health care program is best secured by establishing goals of care, defining desired outcomes and identifying high-risk groups requiring particular attention. Careful guidance is required to ensure that health care expenditure delivers on preset objectives. Such investment is likely to be richly rewarded through increases in productivity and improvements in safety alone. ${ }^{3}$ However, blank cheques are never an option, and meeting the challenge of improving sleep health will need to be done within a tight, but not selfdefeating, fiscal framework. For example, given that we recognise that continuous positive airway pressure (CPAP) is a cost-effective therapy for higher-risk and symptomatic obstructive sleep apnoea sufferers, strategies for better targeting care delivery to these groups may allow for much needed and consistent CPAP-funding models across the states and territories.

There is much information already in place to inform practice. For example, guidelines are available on appropriate use of therapeutic devices such as CPAP machines. ${ }^{4}$ However, dissemination of this equipment is unregulated, with the Therapeutic Goods Administration yet to adopt the United States Food and Drug Administration principle of sale of CPAP devices by medical prescription. Guidelines have been recently updated for sleep studies in adults (http://www.sleep.org.au/information/sleep-documents) 


\section{Sleep loss and sleep disorders: key points}

\section{Public health implications of sleep loss: the community burden (page S7)}

- An evaluation of the sleep habits of Australians demonstrates that disrupted sleep, inadequate sleep duration, daytime fatigue, excessive sleepiness and irritability are highly prevalent (20\%-35\%). While about half of these problems are attributable to specific sleep disorders, the balance appears largely due to poor sleep habits or choices to limit sleep opportunity.

- The economic impact of sleep disorders includes costs to Australia of $\$ 5.1$ billion per year of which $\$ 800$ million are direct health care costs of the disorders and of other medical conditions attributable to them, with the balance of $\$ 4.3$ billion mainly attributable to productivity losses and non-health costs of sleep loss-related accidents.

\section{Common sleep disorders affecting individuals and communities}

Obstructive sleep apnoea:

- Obstructive sleep apnoea (OSA) is one of the most common sleep disorders. Population studies using sleep recordings show that OSA affects about 25\% of adult males and $10 \%$ of adult females although most affected individuals do not complain of daytime sleepiness. Combining simple screening questionnaires with home sleep studies is helpful in identifying the severe and symptomatic cases that are likely to benefit most from treatment. Using simplified pathways in controlled studies has shown that patients with a high pretest probability of symptomatic OSA can be managed well in primary care, or by skilled nurses with appropriate specialist sleep service clinical support (page S21).

- Severe OSA is strongly associated with increased mortality, stroke and cardiovascular disease in middle-aged populations. The cardiovascular risk from moderate OSA is uncertain, although the data suggest an increased risk for stroke (particularly in men). There is no evidence of increased cardiovascular risk from mild OSA (page S27).

Shift work disorder (page S17):

- Nearly 1.5 million Australians are employed in shift work. Health, performance and safety are often degraded in shift workers due to the combined effects of circadian rhythm misalignment and inadequate and poor-quality sleep (resulting from disorders such as OSA, insomnia and shift work disorder).

- Optimal rostering, scheduled napping, appropriately timed light and melatonin treatment to promote circadian adaptation, and judicious use of pharmacotherapy are strategies that aim to mitigate the adverse effects of shift work, along with screening for sleep and mood disorders, and close monitoring of risk factors for cardiovascular disease.

Insomnia (page S36):

- Insomnia is a very common disorder, with Australian population surveys showing that $13 \%-33 \%$ of the adult population have regular difficulty either getting to sleep or staying asleep. Chronic insomnia is unlikely to spontaneously remit and, over time, will be characterised by cycles of relapse and remission or persistent symptoms.

- Chronic insomnia is best managed using non-drug strategies such as cognitive behaviour therapy, which can be highly effective. However, if patients have ongoing symptoms there may be a role for adjunctive use of medication such as hypnotics, observing recognised techniques that minimise tolerance and dependency.

Delayed sleep phase disorder (page S16):

- Delayed sleep phase disorder is a circadian rhythm sleep disorder most commonly seen in adolescents. It needs to be differentiated from insomnia - the use of sleep diaries illustrating delayed sleep onset and waking with normal sleep duration, without imposed restriction, confirms the distinction.

- Imposing conventional wake times fails to resolve the phase delay and risks sleep loss and the potential for adverse impact on academic performance and social functioning.

- Awareness and education are important components of the treatment plan. The effects of delayed sleep phase disorder may be minimised by a combination of behavioural and chronotherapeutic strategies. Bright light and melatonin can manipulate the circadian phase; however, their timing in relation to the natural sleep phase is critical to success and sometimes requires specialist input.

Sleep disorders in children (page S37):

Sleep disorders are very common in childhood. They include insufficient sleep, frequent night awakenings and OSA.

- OSA in childhood has important implications for learning, behaviour and cardiovascular health.

- Adenotonsillectomy can be a highly efficacious therapy for paediatric OSA. However, in $20 \%$ of patients, the disease persists despite surgery, particularly among children with obesity, underlying syndromes or malformations.
- an area of potential confusion with the increased availability of diagnostic tools of varying quality in the primary care setting. Accreditation standards among sleep health delivery services, including home and inlaboratory sleep-testing facilities, have led to the evolution an increasingly sophisticated array of benchmarks. These include a recent partnership between the National Association of Testing Authorities and the Australasian Sleep Association to ensure high standards in sleep medicine services (http:// www.nata.com.au; http://www.sleep.org.au/information/sleep-documents). Balancing the principle of optimal standards against accessibility, affordability and viability of services is particularly challenging in Australia, with its unique regional and remote demographics.

The articles in this supplement summarise the current understanding of a range of highly prevalent sleep problems and their impact on individual and community wellbeing. Key learning points from the articles can be found in the Box. The articles outline the burden of common sleep problems and their substantial economic cost in the Australian community. Key bodies such as the Sleep Health Foundation and the Australasian Sleep Association play an important role in articulating these issues to the broader health profession, schools, industry, policymakers and society generally to ensure healthy sleep becomes more of an established community priority. This supplement is an expression of their intent to do so.

Competing interests: Nicholas Antic has received a grant of $\$ 5$ million from Philips Respironics for a large randomised controlled trial of CPAP therapy for obstructive sleep apnoea, with equipment donations from Philips Respironics, ResMed, and Fisher and Paykel. He has received additional equipment donations from ResMed, Philips Respironics and SomnoMed, and lecture fees and payment for development of educational presentations from ResMed. Doug McEvoy has received unconditional grants for sleep research from Philips Respironics and Fisher and Paykel, unconditional equipment grants for research studies from ResMed, Philips Respironics and Air Liquide Australia, and lecture fees from Philips Respironics. Shantha Rajaratnam has served as a consultant through his institution to Vanda Pharmaceuticals, Philips Respironics, EdanSafe, National Transport Commission, Rail, Tram and Bus Union, Australian Workers' Union, Tontine Group, Meda Consumer Healthcare, and has, through his institution, received research grants and unrestricted educational grants from Vanda Pharmaceuticals, Philips Respironics and Cephalon, and reimbursements for conference travel expenses from Vanda Pharmaceuticals. His institution has received equipment donations or other support from Optalert, Compumedics, Philips Lighting and Tyco Healthcare. He has also served as an expert witness and consultant to shift work organisations.

Provenance: Not commissioned; not externally peer reviewed.

1 Bartlett DJ, Marshall NS, Williams A, Grunstein RR. Sleep health New South Wales: chronic sleep restriction and daytime sleepiness. Int Med J 2008; 38: 24-31.

2 Chahal H, Fung C, Kuhle S, Veugelers PJ. Availability and night-time use of electronic entertainment and communication devices are associated with short sleep duration and obesity among Canadian children. Pediatr Obes 2013; 8: 42-51.

3 Deloitte Access Economics. Re-awakening Australia: the economic cost of sleep disorders in Australia, 2010. Canberra, Australia: Deloitte Access Economics, 2011. http://www.sleephealthfoundation.org.au/ pdfs/news/Reawakening\%20Australia.pdf (accessed Sep 2013).

4 Australasian Sleep Association. Position paper: best practice guidelines for provision of CPAP therapy. Version 2.2. 14 Jan 2009. http:// www.sleep.org.au/documents/item/66 (accessed Sep 2013). 